\title{
Treatment of glioblastoma in elderly patients
}

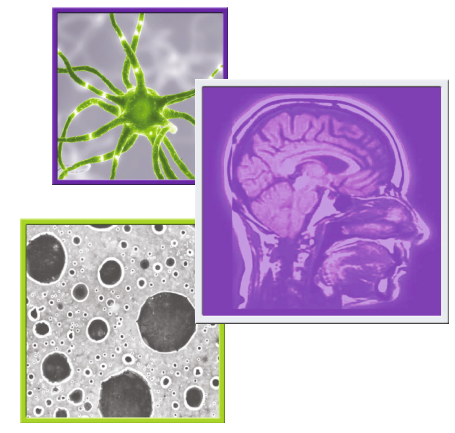

Florian Stockhammer*

\section{PRACTICE POINTS}

- In elderly patients, the treatment strategy should strive for surgical gross total tumor resection.

- If a gross total resection is not feasible, diagnosis should be confirmed by stereotactic biopsy.

- Elderly patients may profit from concomitant and adjuvant temozolomide radiochemotherapy.

- Prospective randomized trials have shown that dose-dense temozolomide is superior to radiotherapy only in glioblastomas with methylated MGMT promoter.

- When temozolomide is not practicable, hypofractionated radiotherapy should be preferred to conventional fractionation.

- Comorbidity is more frequent in elderly patients, limiting temozolomide initiation, but temozolomide toxicity is comparable with adult patients.

SUMMARY: Although glioblastoma occurs mostly in elderly patients, there is a paucity of trials addressing patients older than 70 years of age. Age, by itself, constitutes an unfavorable prognostic factor, which is probably due to unpropitious genetic features, but also due to iatrogenic defeatism. However, many retrospective studies report a survival benefit achieved by aggressive surgical resection seeking gross total removal of contrast-enhancing tumor according to preoperative MRI. Combined radiochemotherapy with concomitant and adjuvant temozolomide has not been investigated in prospective trials. Numerous retrospective studies and a meta-analysis suggest benefit from combined treatment. Prospective randomized trials only evaluated either temozolomide or radiotherapy. Singletreatment hypofractionated radiotherapy performed superior to conventional fractionation. In patients with methylated MGMT promoter, first-line dose-dense temozolomide facilitates prolonged survival. However, there is no comparison with combined radiochemotherapy as the standard-of-care in adult patients. Comorbidity is more frequent in elderly patients, but does not correlate with preterm termination of temozolomide treatment. This review article compiles data proposing a straightforward glioblastoma treatment, irrespective of age.

In adult patients, glioblastoma is the most frequent malignant primary brain tumor, with a peak in the 1970s [1]. Every third patient with newly diagnosed glioblastoma appears to be older than 65 years of age [2]. According to the aging population, we will increasingly deal with glioblastomas in patients of advanced age. However, elderly patients are frequently excluded from clinical trials. But, any cut-off age to consider patients 'elderly' lacks any rationale. The age of 70 years is arbitrary

*Department of Neurosurgery, Universitätsmedizin Göttingen, Robert-Koch-Straße 40, 37075 Göttingen, Germany; Tel.: +49 55139 22804; Fax: +49 3212 1149855; florian.stockhammer@med.uni-goettingen.de

\section{KEYWORDS \\ - elderly patients $\bullet$ glioblastoma • radiotherapy - survival • tumor resection \\ Future Medicine part of}


and not based on any biological events [3]. Thus, considering a patient 'elderly' is inconstant and assigned to the ages of $50,55,60,65$, and, most often, 70 years. The therapy rationale in elderly patients often differs from younger fellows (subsequently called adults) with this diagnosis. Glioblastoma patients of an older age might have a distinct accumulation of genetic changes, naturally reduced life expectancy and less resistance to the tumor or its therapy, causing iatrogenic restriction of treatment intensity $[4,5]$. The latter constitutes a severe bias in retrospective series. In this review, data will be compiled that pre-empt the need for a stigma of the elderly in terms of decision-making.Prognostic factors Age by itself constitutes a dismal prognostic factor in glioblastoma patients [5]. This prognostic role remains within the elderly patients [6], with a median overall survival of just 2.5 months in patients over the age of 75 years [7] or a hazard ratio of 2.64 for shortened survival, when patients exceed the age of 80 years [8].

In glioblastoma patients, methylation of MGMT constitutes a strong prognostic factor in terms of time-to-progression and survival $[9,10]$. In elderly patients, the $M G M T$ promoter is methylated in $43-57.5 \%$, depicting about the same ratio of methylated MGMT promoters compared with patients under the age of 70 years. Deletion of chromosome 10 is associated with dismal prognosis [11] and is specifically found in glioblastoma patients over the age of 70 years [12].

Glioblastomas of elderly patients exhibit frequent alteration in genes regulating VEGF [12]. VEGF expression correlates this WHO grade in gliomas, but does not constitute a prognostic factor within glioblastomas [13]. However, VEGF overexpression serves as treatment target by the anti-VEGF antibody bevacizumab and therefore might constitute a positive predictive feature in the future.

Finally, there are genetic markers associated with a favorable prognosis in glioblastoma patients, which are virtually absent in elderly patients. Mutation of $I D H ~ 1 / 2$ is associated with secondary glioblastomas and a prolonged progressionfree survival [14], but only present in $2 \%$ of elderly patients [15]. A H3F3A G34 mutation, which, in contrast to a $H 3 F 3 A \mathrm{~K} 27$ mutation, is associated with the supratentorial glioblastomas, also constituted a favorable marker, typically found in pediatric but not elderly-type glioblastomas $[15,16]$. Glioma-CpG island methylator phenotype positivity is associated with an overall survival of
150 weeks in contrast to 54 weeks in GliomaCpG island methylator phenotype-negative glioblastoma patients [17], while the median age of the patients is 36 and 59 years, respectively.

Likewise, the adult patients the Karnofsky performance score (KPS) remains a strong prognostic factor in the elderly patients as well $[6,9,18,19]$. General condition and age both influence the treatment decision [20,21], leading to the tendency of less treatment in elderly patients. Thus, the dismal prognosis in elderly patients is prone to be a result of a self-fulfilling prophecy due to defeatism of the physician in charge.

\section{Clinical appearance}

Symptoms and signs of glioblastoma differ according to age. Elderly patients more often present with confusion, memory loss and personality changes, which possibly cause delay of tumor diagnosis [5,6]. Seizures, as an initial symptom of malignant brain tumors, appear to be rare in elderly patients, which might be due a dominance of primary glioblastomas from 71 to 80 years of age [22]. Seizure is more common in WHO grade II and III gliomas [23], which then progress to secondary glioblastoma, and were more likely to occur from 51 to 60 years of age. According to genetics, secondary glioblastoma are associated with TP53 and icocitratdehydrogenase 1 or 2 mutations. The latter, which is associated with seizure as an initial symptom in low-grade glioma [24], is rare in the elderly, providing an explanation for fewer seizures [15]. Concerning the general condition, elderly patients initially present with a lower KPS compared with adult patients [25].

\section{Surgery}

Despite advances in tumor resection, there is no surgical cure for glioblastoma patients, which is due to infiltrative growth of these tumors. However, maximum cytoreductive surgery has been shown to prolong survival in glioblastoma patients $[26,27]$. For complete resection of the contrast-enhancing tumor, fluorescence-guided resection has proven to be beneficial, being the current gold standard $[28,29]$. Thus, fluorescenceguided resection often exceeds the contrastenhancing tumor [30]. However, in some series, elderly patients receive more biopsies than resection $[25,31]$, revealing a strong confounder due to individual treatment aggressiveness in various centers or trials. 
To elucidate the role of resection in elderly patients suffering from glioblastoma, a variety of retrospective studies have been conducted. In an analysis of 40 matched pairs of elderly patients receiving either resection or biopsy, the median survival was 5.7 and 4.0 months, respectively [32]. This results in a significant survival benefit due to the more aggressive treatment, but with equal perioperative morbidity. Of note, the relatively short survival time in this study might be due the fact that only $10 \%$ of the patients received temozolomide. A median survival of 17.4 and 11.4 months could be achieved in elderly patients receiving gross and subtotal resection, respectively [25]. Although $40 \%$ of the patients underwent combined radiochemotherapy, the brisk difference in survival of the resected patients by almost 1 year recalls the obvious strong bias in retrospective studies. Ewelt and coworkers also report a median survival of 13.9 months after gross total resection, 7.0 months after partial resection and 2.2 months following biopsy [7]. A multivariate analysis including age and performance status confirmed the prognostic value of resection, but, considering the extreme survival difference, a treatment confounder must be assumed again. Likewise, survival times of 18 , 16 and 6 months according to gross total resection, partial resection and biopsy, respectively, are reported in another study [33]. In all these publications, the median survival of patients receiving a biopsy were only 6 months at best, raising the question of whether biopsy should have been performed at all. However, there are good reasons to overcome the defeatism that omits any diagnostic efforts when patients are not candidates for gross total tumor resection. First, despite typical appearance of glioblastoma, differential diagnosis, such as metastasis, lymphoma or rare entities, should be kept in mind when treatment decisions in elderly patients will be made [5,34]. Second, stereotactic biopsy is a very low-risk procedure, which can easily be performed under local anesthesia [35]. Third, even genetic markers, such as MGMT promoter methylation, can be determined on serial stereotactic specimens [36].

On the other hand, performing a tumor resection provides the opportunity for additional local treatment. In a retrospective matched-pair study of elderly patients receiving intraoperative placement of carmustine wafers, the patients had a longer survival compared with equally treated patients without interstitial chemotherapy (8.7 vs 5.5 months, respectively) [37]. However, the benefit seems to be restricted to only complete resections in patients with a good KPS [38]. As the carmustine wafer implantation harbors an up to fivefold-increased risk for severe complications such as edema, postoperative seizures, cerebrospinal fluid leakage or infections in a dose-dependent manner [39], this treatment must be critically evaluated for the individual patient.

\section{Radiotherapy}

Radiotherapy was the former standard therapy in elderly glioblastoma patients and is superior to supportive care [40]. Patients are mostly assigned to conventional radiotherapy with 30 fractions of 1.8-2 Gy. Alternatively, abbreviated protocols have been introduced to meet the needs of patients with expected shortened survival. Due to hypofractionation, the radiation was completed within 2, 3 or 4 weeks, applying 30 , 45 and $50 \mathrm{~Gy}$, respectively. Hypofractionation revealed equal survival compared with the conventional 60 Gy in 6 weeks [41-43]. In the Nordic elderly trial, temozolomide with $200 \mathrm{mg} / \mathrm{m}^{2}$ was administered as a third arm and performed superiorly to conventional but equally to hypofractionated radiotherapy [43]. Likewise for NOA-08, the scope of the Nordic elderly trial was less aggressive treatment considering the poorer prognosis in elderly patients. Thus, until recurrence, neither concomitant nor sequential treatment was offered. In fact, the treatment was mostly discontinued due to tumor progression rather than toxicity.

\section{Concomittant \& adjuvant chemotherapy}

The EORTC trial 26981/22981 introducing a combined concomitant and adjuvant administration of temozolomide provided class 1 evidence for survival benefit $[44,45]$. However, due to the conviction that elderly patients could not be referred to such intensive treatment, patients older than 70 years of age were excluded from the trial. Likewise, resent prospective trials for elderly glioblastoma patients sought less intense and toxic treatment without offering a combined treatment arm $[43,46]$.

Doubt regarding an effect of concomitant and adjuvant temozolomide in elderly patients was raised when recursive partitioning analysis of EORTC 26981/22981 was performed. Those older than 50 years qualify for group IV or V, the latter group showing a nonsignificant benefit from combined treatment. Disregarding the 
fact that no resection had been performed and that a poor performance status was also a criterion for group $\mathrm{V}$, it was presumed that older age tended to be a predictive factor for combined treatment being less effective [47]. However, a later long-term follow-up subgroup analysis of the same study cohort reports a survival benefit of patients older than 60 years of age treated with combined therapy (Table 1) [45]. These data are in line with retrospective matched-pair data revealing a benefit from combined radiochemotherapy, disregarding age as a restricting factor, with a hazard ratio of 3.1 [19]. Likewise, prospective data of patients of ages between 60 and 75 years reported a prolonged median survival of 16.3 months when treated with radiochemotherapy compared with 11.2 months with radiation only [48]. The scope of this study was the safety of fluorescence-guided resection; therefore, none of the patients had biopsies only. Furthermore, patients in altered general condition were excluded. This selection might explain the superior survival data. Vast retrospective data come from 13,932 patients with glioblastoma compare the era before and after the introduction of radiotherapy combined with temozolomide [49]. However, there were no records of the chemotherapy actually administered, so the treatment was assumed according to the era the treatment took place. On the other hand, before temozolomide became standard treatment, nitrosurea was commonly administered in patients with high-grade glioma. However, in elderly patients, temozolomide proved to be superior compared with radiotherapy alone or adjuvant procarbacin, vincristine and lomustine treatment, as shown by a prospective series with consecutively treated patient groups [18].

Other retrospective smaller series report a benefit of combined treatment compared with radiotherapy only in elderly patients. However, these studies restricted inclusion criteria to good general state, excluded patients who only received biopsies and allowed a selection bias by omitting multivariate or matched-pair analysis [21,51]. As a consequence, a meta-analysis of these studies addressing the benefit of combined radiochemotherapy compared with radiotherapy alone proclaims class 2a evidence for clear survival benefit due to combined treatment [52].

Prospective controlled trials dealing solely with elderly patients randomized radiotherapy alone versus dose-dense temozolomide as single first-line treatment (NOA-08 trial) [46]. For the entire study, the temozolomide treatment cohort appeared not to be inferior. Benefit was shown for the subgroup of patients with methylated MGMT promoter receiving temozolomide. However, concerning the actual treatment recommendation in elderly patients, NOA-08 raises questions:

- NOA-08 has had no arm offering combined radiochemotherapy, which is actually the

Table 1. Controlled studies of elderly patients treated first-line for glioblastoma by combined concomittant and adjuvant radiochemotherapy.

\begin{tabular}{|c|c|c|c|c|c|c|c|}
\hline $\begin{array}{l}\text { Study } \\
\text { (year) }\end{array}$ & Design & $\begin{array}{l}\text { Age } \\
\text { restriction } \\
\text { (years) }\end{array}$ & $\mathrm{n}$ & $\begin{array}{l}\text { Median survival/ } \\
\text { age of radiotherapy } \\
\text { group (years) }\end{array}$ & $\begin{array}{l}\text { Median survival/age of } \\
\text { combined treatment } \\
\text { group (years) }\end{array}$ & $\begin{array}{l}\mathrm{HR}(95 \% \mathrm{Cl}) / \mathrm{p} \text {-value (log } \\
\text { rank) for comparison } \\
\text { with adult patients }\end{array}$ & Ref. \\
\hline \multirow{3}{*}{$\begin{array}{l}\text { Behm et al. } \\
\text { (2013) }\end{array}$} & Retrospective & $>65$ & 90 & $3.6 / 71.9$ & $8.7 / 70.9$ & $3.1(1.92-4.99) / p<0.0001$ & \multirow[t]{3}{*}[19]{} \\
\hline & Subgroup & $>70$ & 62 & $3.2 / 73.6$ & $7.5 / 73.0$ & $4.5(2.42-8.14) / \mathrm{p}<0.0001$ & \\
\hline & Matched pair & $>75$ & 24 & $3.2 / 77.4$ & $9.2 / 76.2$ & $9.0(3.04-26.9) / \mathrm{p}<0.0001$ & \\
\hline $\begin{array}{l}\text { Stupp et al. } \\
\text { (2009) }\end{array}$ & $\begin{array}{l}\text { Prospective, } \\
\text { randomized, multivariate }\end{array}$ & $>60-70$ & 170 & $11.8 / \mathrm{nr}$ & $10.9 / \mathrm{nr}$ & $1.4(1.03-2.00) / \mathrm{nr}$ & {$[45]$} \\
\hline $\begin{array}{l}\text { Stummer } \\
\text { et al. (2011) }\end{array}$ & $\begin{array}{l}\text { Prospective, subgroup, } \\
\text { multivariate }\end{array}$ & $\geq 60-75$ & 130 res & $11.2 / 66.0$ & $16.3 / 68.0$ & $\mathrm{nr} / \mathrm{p}=0.0145$ & [48] \\
\hline \multirow{2}{*}{$\begin{array}{l}\text { Johnson } \\
\text { and O'Neill } \\
\text { (2012) }\end{array}$} & Retrospective & $\geq 60-69$ & 2334 & $10.2^{\dagger} / \mathrm{nr}$ & $13.0^{\dagger} / \mathrm{nr}$ & $\mathrm{nr} / \mathrm{p}<0.0001$ & \multirow[t]{2}{*}{ [49] } \\
\hline & Historical, multivariate & $\geq 70-79$ & 1549 & $7.9^{\dagger} / \mathrm{nr}$ & $9.3^{+} / \mathrm{nr}$ & $\mathrm{nr} / \mathrm{p}<0.0001$ & \\
\hline $\begin{array}{l}\text { Ewelt et al. } \\
\text { (2011) }\end{array}$ & Retrospective, selected & $>65$ & 72 & $4.5 / 70.6$ & $15.0 / 68.5$ & $\mathrm{nr} / \mathrm{nr}$ & {$[7]$} \\
\hline \multirow{2}{*}{$\begin{array}{l}\text { Arrigo } \\
\text { et al. (2012) }\end{array}$} & Prospective & \multirow[t]{2}{*}{$>70$} & 129 res & $4.4 / \mathrm{nr}$ & $5.5 / \mathrm{nr}$ & $\mathrm{nr} / \mathrm{nr}$ & \multirow[t]{2}{*}{ [50] } \\
\hline & Subgroup, multivariate & & $89 \mathrm{bx}$ & $5.8 / \mathrm{nr}$ & $13.3 / \mathrm{nr}$ & $\mathrm{nr} / \mathrm{nr}$ & \\
\hline
\end{tabular}


standard therapy in adult patients. Therefore, even in patients with methylated MGMT promoter receiving temozolomide, omitting concomitant radiotherapy could result in undertreatment;

- MGMT data are mostly missing in patients who received stereotactic biopsy, which is comparatively frequent in elderly patients;

- In many retrospective reports, combined treatment revealed a clear benefit without stratification for MGMT status [7,9,19,33,40,48,50,51] .

This suggests a high effect of combined treatment, which could be even stronger when restricted to patients with methylated MGMT promoter, anticipating a better effect of combined treatment compared with temozolomide only. This was shown in a large multicenter cohort study, where 233 elderly patients with glioblastoma were treated with radiotherapy, temozolomide or combined treatment [10]. In tumors with methylated MGMT promoter, application of temozolomide was correlated with prolonged survival. Combined treatment in nonmethylated MGMT promoter glioblastoma did not show a significant survival benefit. However, the latter analysis was based on 66 patients only. As the methylation status was not blinded for treatment decision, there could have been a bias by omitting temozolomide in nonmethylated patients [53]. In fact, the survival curves of methylated and nonmethylated MGMT promoters do not cross over [10], leaving the possibility of type II error on the basis of a slight temozolomide effect. A meta-analysis of these trials, offering only temozolomide or radiotherapy as first-line treatment, concludes with a recommendation of temozolomide treatment for patients with methylated MGMT promoter. Although randomized trials are welcome, as they provide high-class evidence, there are caveats due to the trial protocols, as already critically reviewed by Fiorentino et al. [54]:

- The Nordic trial included patients from the age of 60 years, conflicting initial EORTC data;

- NOA-08 also included patients with a very low KPS, providing a serious confounder;

- NOA-08 also includes anaplastic gliomas;

- Temozolomide regimes differed from standard high dose over 5 days to intermediate daily dose, applied one week on/one week off;
- Salvage treatment was different, with crossover designs, not giving a true control treatment.

According to the meta-analysis of firstline single treatment, interestingly, temozolomide performed slightly superiorly in all patients, with a hazard ratio of $0.86(95 \% \mathrm{CI}$ : 0.75-1.0). Thus, temozolomide might not be without an effect in nonmethylated MGMT promoter glioblastomas. Besides methylation of the promoter, MGMT activity can also be altered due to loss of heterozygosity of the MGMT gene or the entire deletion of chromosome $10 \mathrm{q}$. The latter is highly associated with glioblastomas in the elderly [12]. As mentioned above, $10 \mathrm{q}$ deletion is linked to dismal prognosis [11]; however, when treated with temozolomide, these patients benefit significantly [55]. Interestingly, the favorable impact of $10 \mathrm{q}$ deletion and temozolomide treatment was emphasized in elderly patients [55]. This underlines the role of temozolomide in elderly patients. A meta-analysis of studies comparing temozolomide with radiotherapy as a single first-line treatment favors temozolomide, with a borderline improvement of overall survival [56].

The combination of temozolomide with hypofractionated radiotherapy could accomplish both an intense treatment and a concise radiation protocol. This has been prospectively addressed by Muni et al., reporting a survival advantage in poor-performing elderly or dependent patients [57]. Adjuvant temozolomide was added to hypofractionated radiation, reaching a median survival of 9.4 compared with 7.3 months, when only radiation was performed. Currently, EORTC 26062/22061 addresses the issue of combined hypofractionated treatment, enrolling elderly patients only. Preliminary data applying this protocol exist from a series of 31 patients, of which 16 were older than 70 years [58]. Tolerance was good, with unexpectedly low toxicity. Hypofractionatad radiotherapy with 40 Gy in 15 fractions with concomitant temozolomide followed by adjuvant cycles, including 71 patients over the age of 70 years, was reported by Minniti et al. [59]. The median overall and progression-free survival was 12.4 and 6 months, respectively. However, efficiency data of concomitant temozolomide in abbreviated radiotherapy do not exist and might prove temozolomide to only be equal to adjuvant 
therapy, as retrospective data suggests [60]. Perhaps lowered total concomitant temozolomide dose due to the abbreviated radiotherapy hampers additional efficiency. Other than for hypofractionated radiotherapy, there is no trial planned addressing elderly patients receiving the current standard-treatment protocol.

It was assumed that older patients reveal enhanced toxicity when treated with alkylating agents. In a series of 19 glioblastoma patients older than 65 years of age, severe myelosuppression was seen in $15 \%$ [61]. The total toxicity rate was reported at $42 \%$, including inappropriate $\mathrm{ADH}$ secretion and fatigue, which is not necessarily related to temozolomide administration. Likewise, only $14 \%$ of the elderly patients had to terminate temozolomide due to myelosuppression [19], similar to $12 \%$ of the patients after concomitant therapy in the EORTC trial [44]. A series of 43 patients older than 65 years of age receiving concomitant temozolomide reports termination of temozolomide due to myelosuppression in only one patient (2\%) [33]. Three further patients stopped temozolomide due to pneumonia or rash, resulting in a toxicity rate of $9 \%$. No patient had to stop temozolomide due to toxicity in a prospective trial omitting radiation and applying temozolomide only on days $1-5$ in a 28-day cycle [31]. There was also no additional toxicity in elderly patients reported by Oszvald and coworkers [25]. CTC grade 3 and 4 thrombocytopenia or neutropenia were reported in 12.5 and $5.4 \%$ of patients over the age of 65 years, and 11.5 and $3.8 \%$ over the age of 70 years, respectively [62]. These findings were no different to those of glioblastoma patients under the age of 65 years [62]. These data, compiled in Table 2, report a comparable toxicity with that known in patients younger than 65 years of age $[44,63]$. Of note, similar toxicity was considered as serious in the trials omitting radiotherapy, questioning the role of temozolomide [56].

Obviously comorbidity appears more likely in elderly than adult patients. This comorbidity by itself is accountable for worse prognosis. However, when comorbidity did not provide contraindication for temozolomide initiation, there was no association with toxicity-related termination of temozolomide [67].

\section{Quality of life}

Besides survival and toxicity, the patient's quality of life and self-dependence must be kept in mind. In EORTC 26981/22981, the rate of patients reporting fatigue was increased from 26 to $33 \%$ when radiotherapy was combined with temozolomide [44]. The increase of fatigue also occurred in concomitant temozolomide applying an abbreviated radiotherapy over 3 weeks [59]. Besides fatigue, the latter study also reported improvement in all functioning and symptom scores. However, as only half of the patients completed the questionnaire after 6 months, selection must be assumed. In elderly patients, radiotherapy implies the risk of cognitive decline. Compared with temozolomide treatment, patients suffered from communication deficit [46] and a potential decline of cognitive, physical and role functioning [43]. On the other hand, tumor progression is the major cause for decline in quality of life [68], thus offering the most effective treatment might provide quality of life maintenance as well. Thus, elderly patients must be closely watched and considered for sparing from intense treatment, such as completing radiation, in therapy-related deterioration.

Table 2. Hematologic toxicity in patinets treated by concomittant and adjuvant radiochemotherapy.

\begin{tabular}{|c|c|c|c|c|c|c|}
\hline Study (year) & $\begin{array}{l}\text { Age } \\
\text { restriction } \\
\text { (years) }\end{array}$ & $\mathrm{n}$ & $\begin{array}{l}\text { Neuropenia CTC } \\
\text { grade } 3 / 4, \% \\
\text { (patients [n]) }\end{array}$ & $\begin{array}{l}\text { Thrombopenia CTC } \\
\text { grade } 3 / 4, \% \\
\text { (patients [n]) }\end{array}$ & $\begin{array}{l}\text { Toxicity-related temozolomide } \\
\text { termination (\%) }\end{array}$ & Ref. \\
\hline Fiorentino et al. (2013) & $>65$ & 35 & 0 & $11.4(4)$ & $\mathrm{nr}$ & [64] \\
\hline Behm et al. (2013) & $>70$ & 56 & $1.7(1)$ & $14.3(8)$ & 14 & [19] \\
\hline Sijben et al. (2008) & $>65$ & 19 & $15.7(3)$ & $15.7(3)$ & 26 & [61] \\
\hline Combs et al. (2008) & $>65$ & 43 & $2.3(1)$ & $2.3(1)$ & 11.6 & [33] \\
\hline Oszvald et al. (2012) & $>65$ & 58 & $\mathrm{nr}$ & $\mathrm{nr}$ & 8.6 & [25] \\
\hline Minniti et al. (2008) & $>70$ & 32 & $12.5(4)$ & $21.8(7)$ & 6.5 & [65] \\
\hline Lee et al. (2013) & $>70$ & 20 & $10(2)$ & $10(2)$ & $\mathrm{nr}$ & [66] \\
\hline Balducci et al. (2012) & $>65$ & 56 & $1.7(1)$ & $3.4(2)$ & $\mathrm{nr}$ & [62] \\
\hline
\end{tabular}




\section{Conclusion \& future perspective}

In general, patients with glioblastoma should be treated irrespective of age with gross total resection and combined radiochemotherapy. The predictive role of $M G M T$ promoter methylation must be kept in mind when patients reveal risk factors for temozolomide application. The role of first-line bevacizumab treatment is a matter of current trials. As proangiogenic proteins such as VEGF seem to play a paramount role in glioblastoma in elderly patients, bevacizumab is likely to give additional benefit. However, the patient's exposure to extra risk must be critically evaluated. In

\section{References}

Papers of special note have been highlighted as:

- of interest

-• of considerable interest

1 Fisher JL, Palmisano S, Schwartzbaum JA, Svensson T, Lonn S. Comorbid conditions associated with glioblastoma. J. Neurooncol. 116, 585-591 (2014).

2 Dolecek TA, Propp JM, Stroup NE, Kruchko C. CBTRUS statistical report: primary brain and central nervous system tumors diagnosed in the United States in 2005-2009. Neuro Oncol. 14(Suppl. 5), v1-v49 (2012).

3 Yovino S, Grossman SA. Treatment of glioblastoma in "elderly" patients. Curr. Treat. Options Oncol. 12, 253-262 (2011).

4 Lutterbach J, Bartelt S, Momm F, Becker G, Frommhold H, Ostertag C. Is older age associated with a worse prognosis due to different patterns of care? A long-term study of 1346 patients with glioblastomas or brain metastases. Cancer 103, 1234-1244 (2005).

5 Lowry JK, Snyder JJ, Lowry PW. Brain tumors in the elderly: recent trends in a Minnesota cohort study. Arch. Neurol. 55, 922-928 (1998).

6 Chaichana KL, Chaichana KK, Olivi A et al. Surgical outcomes for older patients with glioblastoma multiforme: preoperative factors associated with decreased survival. Clinical article. J. Neurosurg. 114, 587-594 (2011).

7 Ewelt C, Goeppert M, Rapp M, Steiger HJ, Stummer W, Sabel M. Glioblastoma multiforme of the elderly: the prognostic effect of resection on survival. J. Neurooncol. 103, 611-618 (2011).

8 Konglund A, Helseth R, Lund-Johansen M, Helseth E, Meling TR. Surgery for high-grade gliomas in the aging. Acta Neurol. Scand. 128, 185-193 (2013).

the future, clinical trials should omit an upper age exclusion criterion to reveal the age-related reality in this tumor entity.

\section{Financial \& competing interests disclosure}

The author has no relevant affliations or financial involvement with any organization or entity with a financial interest in or financial conflict with the subject matter or materials discussed in the manuscript. This includes employment, consultancies, honoraria, stock ownership or options, expert testimony, grants or patents received or pending, or royalties.

No writing assistance was utilized in the production of this manuscript.

9 Brandes AA, Franceschi E, Tosoni A et al. Temozolomide concomitant and adjuvant to radiotherapy in elderly patients with glioblastoma: correlation with $M G M T$ promoter methylation status. Cancer 115 , 3512-3518 (2009).

10 Reifenberger G, Hentschel B, Felsberg J et al. Predictive impact of $M G M T$ promoter methylation in glioblastoma of the elderly. Int. J. Cancer 131, 1342-1350 (2012).

- Comprehensive multicenter observational study investigating $M G M T$ promoter methylation in 233 elderly patients with progression and survival data.

11 Tada K, Shiraishi S, Kamiryo T et al. Analysis of loss of heterozygosity on chromosome 10 in patients with malignant astrocytic tumors: correlation with patient age and survival. J. Neurosurg. 95, 651-659 (2001).

12 Bozdag S, Li A, Riddick G et al. Age-specific signatures of glioblastoma at the genomic, genetic, and epigenetic levels. PLoS ONE 8, e62982 (2013).

13 Oehring RD, Miletic M, Valter MM et al. Vascular endothelial growth factor (VEGF) in astrocytic gliomas - a prognostic factor? J. Neurooncol. 45, 117-125 (1999).

14 Weller M, Felsberg J, Hartmann C et al. Molecular predictors of progression-free and overall survival in patients with newly diagnosed glioblastoma: a prospective translational study of the German Glioma Network. J. Clin. Oncol. 27, 5743-5750 (2009).

- Current genetic markers of interest investigated in 126 malignant gliomas.

15 Wiestler B, Claus R, Hartlieb SA et al. Malignant astrocytomas of elderly patients lack favorable molecular markers: an analysis of the NOA-08 study collective. Neuro Oncol. 15, 1017-1026 (2013).

16 Sturm D, Witt H, Hovestadt V et al. Hotspot mutations in $H 3 F 3 A$ and $I D H I$ define distinct epigenetic and biological subgroups of glioblastoma. Cancer Cell 22, 425-437 (2012).

17 Noushmehr H, Weisenberger DJ, Diefes K et al. Identification of a CPG island methylator phenotype that defines a distinct subgroup of glioma. Cancer Cell 17, 510-522 (2010).

18 Brandes AA, Vastola F, Basso U et al. A prospective study on glioblastoma in the elderly. Cancer 97, 657-662 (2003).

19 Behm T, Horowski A, Schneider S et al. Concomitant and adjuvant temozolomide of newly diagnosed glioblastoma in elderly patients. Clin. Neurol. Neurosurg. 115, 2142-2146 (2013).

20 Barker FG 2nd, Chang SM, Larson DA et al. Age and radiation response in glioblastoma multiforme. Neurosurgery 49, 1288-1297; discussion 1297-1288 (2001).

21 Abhinav K, Aquilina K, Gbejuade H, La M, Hopkins K, Iyer V. A pilot study of glioblastoma multiforme in elderly patients: treatments, O-6-methylguanine-DNA methyltransferase (MGMT) methylation status and survival. Clin. Neurol. Neurosurg. 115, 1375-1378 (2013).

22 Ohgaki H, Dessen P, Jourde B et al. Genetic pathways to glioblastoma: a population-based study. Cancer Res. 64, 6892-6899 (2004).

23 van Breemen MS, Wilms EB, Vecht CJ. Epilepsy in patients with brain tumours: epidemiology, mechanisms, and management. Lancet Neurol. 6, 421-430 (2007).

24 Stockhammer F, Misch M, Helms HJ et al. IDH1/2 mutations in WHO grade II astrocytomas associated with localization and seizure as the initial symptom. Seizure 21, 194-197 (2012).

25 Oszvald A, Guresir E, Setzer M et al. Glioblastoma therapy in the elderly and the importance of the extent of resection 
regardless of age. J. Neurosurg. 116, 357-364 (2012).

26 Stummer W, Reulen HJ, Meinel T et al. Extent of resection and survival in glioblastoma multiforme: identification of and adjustment for bias. Neurosurgery 62 , 564-576; discussion 564-576 (2008).

27 Pichlmeier U, Bink A, Schackert G, Stummer W. Resection and survival in glioblastoma multiforme: an RTOG recursive partitioning analysis of ALA study patients. Neuro Oncol. 10, 1025-1034 (2008).

28 Hefti M. Fluorescence-guided surgery for brain tumors. CNS Oncol. 2, 67-74 (2013).

29 Stummer W, Pichlmeier U, Meinel T, Wiestler OD, Zanella F, Reulen HJ.

Fluorescence-guided surgery with 5-aminolevulinic acid for resection of malignant glioma: a randomised controlled multicentre Phase III trial. Lancet Oncol. 7, 392-401 (2006)

-. The 5-ALA trial introducing fluorescenceguided resection in glioblastomas. The complete resection of contrast-enhancing tumor can be achieved in two-thirds of patients using fluorescence, while with the conventional technique, only one-third of patients had complete resection. 6-month progression-free survival is improved after fluorescence-guided resection.

30 Stockhammer F, Misch M, Horn P, Koch A, Fonyuy N, Plotkin M. Association of F18-fluoro-ethyl-tyrosin uptake and 5 -aminolevulinic acid-induced fluorescence in gliomas. Acta Neurochir. (Wien) 151, 1377-1383 (2009).

31 Gallego Perez-Larraya J, Ducray F, Chinot O et al. Temozolomide in elderly patients with newly diagnosed glioblastoma and poor performance status: an ANOCEF Phase II trial. J. Clin. Oncol. 29, 3050-3055 (2011).

32 Chaichana KL, Garzon-Muvdi T, Parker S et al. Supratentorial glioblastoma multiforme: the role of surgical resection versus biopsy among older patients. Ann. Surg. Oncol. 18, 239-245 (2011).

33 Combs SE, Wagner J, Bischof M et al. Postoperative treatment of primary glioblastoma multiforme with radiation and concomitant temozolomide in elderly patients. Int. J. Radiat. Oncol. Biol. Phys. 70, 987-992 (2008).

34 Kamp MA, Santacroce A, Zella $S$ et al. Is it a glioblastoma? In dubio pro 5-ALA! Acta Neurochir. (Wien) 154, 1269-1273 (2012).

35 Kreth FW, Muacevic A, Medele R, Bise K, Meyer T, Reulen HJ. The risk of haemorrhage after image guided stereotactic biopsy of intra-axial brain tumours - a prospective study. Acta Neurochir. (Wien) 143, 539-545; discussion 545-536 (2001).

36 Grasbon-Frodl EM, Kreth FW, Ruiter M et al. Intratumoral homogeneity of $M G M T$ promoter hypermethylation as demonstrated in serial stereotactic specimens from anaplastic astrocytomas and glioblastomas. Int. J. Cancer 121, 2458-2464 (2007).

37 Chaichana KL, Zaidi H, Pendleton C et al. The efficacy of carmustine wafers for older patients with glioblastoma multiforme: prolonging survival. Neurol. Res. 33, 759-764 (2011).

38 Menei P, Metellus P, Parot-Schinkel E et al. Biodegradable carmustine wafers (Gliadel) alone or in combination with chemoradiotherapy: the French experience. Ann. Surg. Oncol. 17, 1740-1746 (2010).

39 De Bonis P, Anile C, Pompucci A et al. Safety and efficacy of Gliadel wafers for newly diagnosed and recurrent glioblastoma. Acta Neurochir. (Wien) 154, 1371-1378 (2012).

40 Keime-Guibert F, Chinot O, Taillandier L et al. Radiotherapy for glioblastoma in the elderly. N. Engl. J. Med. 356, 1527-1535 (2007).

41 Roa W, Brasher PM, Bauman G et al. Abbreviated course of radiation therapy in older patients with glioblastoma multiforme: a prospective randomized clinical trial. J. Clin. Oncol. 22, 1583-1588 (2004).

42 Chang EL, Yi W, Allen PK, Levin VA, Sawaya RE, Maor MH. Hypofractionated radiotherapy for elderly or younger lowperformance status glioblastoma patients: outcome and prognostic factors. Int. J. Radiat. Oncol. Biol. Phys. 56, 519-528 (2003).

43 Malmstrom A, Gronberg BH, Marosi C et al. Temozolomide versus standard 6-week radiotherapy versus hypofractionated radiotherapy in patients older than 60 years with glioblastoma: the Nordic randomised, Phase 3 trial. Lancet Oncol. 13, 916-926 (2012).

44 Stupp R, Mason WP, van den Bent MJ et al. Radiotherapy plus concomitant and adjuvant temozolomide for glioblastoma. N. Engl. J. Med. 352, 987-996 (2005).

-. Prospective Phase III trial introducing temozolomide as primary concomitant and adjuvant treatment for glioblastoma.

45 Stupp R, Hegi ME, Mason WP et al. Effects of radiotherapy with concomitant and adjuvant temozolomide versus radiotherapy alone on survival in glioblastoma in a randomised Phase III study: 5-year analysis of the EORTC-NCIC trial. Lancet Oncol. 10, 459-466 (2009)
46 Wick W, Platten M, Meisner C et al. Temozolomide chemotherapy alone versus radiotherapy alone for malignant astrocytoma in the elderly: the NOA-08 randomised, Phase 3 trial. Lancet Oncol. 13, 707-715 (2012).

-. Prospective Phase III trial showing noninferiority of temozolomide as primary treatment for malignant glioma in elderly patients. In methylated $M G M T$ promoter patients, temozolomide is associated with prolonged survival. However, no combined treatment arm is designed.

47 Mirimanoff RO, Gorlia T, Mason W et al. Radiotherapy and temozolomide for newly diagnosed glioblastoma: recursive partitioning analysis of the EORTC 26981/22981-NCIC CE3 Phase III randomized trial. J. Clin. Oncol. 24, 2563-2569 (2006).

48 Stummer W, Nestler U, Stockhammer F et al. Favorable outcome in the elderly cohort treated by concomitant temozolomide radiochemotherapy in a multicentric Phase II safety study of 5-ALA. J. Neurooncol. 103, 361-370 (2011).

49 Johnson DR, O’Neill BP. Glioblastoma survival in the United States before and during the temozolomide era. J. Neurooncol. 107, 359-364 (2012).

50 Arrigo RT, Boakye M, Skirboll SL. Patterns of care and survival for glioblastoma patients in the Veterans population. J. Neurooncol. 106, 627-635 (2012)

51 Mariniello G, Peca C, del Basso de Caro M, Giamundo A, Donzelli R, Maiuri F. Glioblastoma in the elderly: the impact of the advanced age on treatment and survival. J. Neurol. Surg. A Cent. Eur. Neurosurg. doi:10.1055/s-0033-1349713 (2014) (Epub ahead of print).

52 Yin AA, Zhang LH, Cheng JX et al. Radiotherapy plus concurrent or sequential temozolomide for glioblastoma in the elderly: a meta-analysis. PLoS ONE 8, e74242 (2013).

-. Meta-analysis resulting in a clear benefit of elderly patients receiving combined first-line treatment. As this treatment was not included in the randomized controlled trials, this paper argues for combined treatment, irrespective of age.

53 Hegi ME, Diserens AC, Gorlia T et al. $M G M T$ gene silencing and benefit from temozolomide in glioblastoma. N. Engl. J. Med. 352, 997-1003 (2005).

54 Fiorentino A, De Bonis P, Chiesa S, Balducci M, Fusco V. Elderly patients with glioblastoma: the treatment challenge. Expert Rev. Neurother. 13, 1099-1105 (2013). 
55 Wemmert S, Ketter R, Rahnenfuhrer J et al. Patients with high-grade gliomas harboring deletions of chromosomes $9 \mathrm{p}$ and $10 \mathrm{q}$ benefit from temozolomide treatment. Neoplasia 7 , 883-893 (2005).

- Unique investigation showing the paradoxical benefit of $10 q$ deletion when temozolomide has been applied, which applies to elderly patients in particular.

56 Yin AA, Cai S, Dong Y et al. A meta-analysis of temozolomide versus radiotherapy in elderly glioblastoma patients. J. Neurooncol. 116, 315-324 (2014).

57 Muni R, Minniti G, Lanzetta G et al. Short-term radiotherapy followed by adjuvant chemotherapy in poor-prognosis patients with glioblastoma. Tumori 96, 60-64 (2010).

58 Reyngold M, Lassman AB, Chan TA, Yamada Y, Gutin PH, Beal K. Abbreviated course of radiation therapy with concurrent temozolomide for high-grade glioma in patients of advanced age or poor functional status. J. Neurooncol. 110, 369-374 (2012).

59 Minniti G, Scaringi C, Baldoni A et al. Health-related quality of life in elderly patients with newly diagnosed glioblastoma treated with short-course radiation therapy plus concomitant and adjuvant temozolomide. Int. J. Radiat. Oncol. Biol. Phys.86, 285-291 (2013).

60 Cao JQ, Fisher BJ, Bauman GS, Megyesi JF, Watling CJ, Macdonald DR.

Hypofractionated radiotherapy with or without concurrent temozolomide in elderly patients with glioblastoma multiforme: a review of ten-year single institutional experience. J. Neurooncol.107, 395-405 (2012).

61 Sijben AE, McIntyre JB, Roldan GB et al. Toxicity from chemoradiotherapy in older patients with glioblastoma multiforme. J. Neurooncol. 89, 97-103 (2008).

62 Balducci M, Fiorentino A, De Bonis $\mathrm{P}$ et al. Impact of age and co-morbidities in patients with newly diagnosed glioblastoma: a pooled data analysis of three prospective monoinstitutional Phase II studies. Med. Oncol. 29, 3478-3483 (2012).

63 Hau P, Koch D, Hundsberger T et al. Safety and feasibility of long-term temozolomide treatment in patients with high-grade glioma. Neurology 68, 688-690 (2007).
64 Fiorentino A, Chiumento C, Caivano R, Cozzolino M, Pedicini P, Fusco V. Adjuvant radiochemotherapy in the elderly affected by glioblastoma: single-institution experience and literature review. Radiol. Med. 118, 870-881 (2013).

65 Minniti G, De Sanctis V, Muni R et al. Radiotherapy plus concomitant and adjuvant temozolomide for glioblastoma in elderly patients. J. Neurooncol. 88, 97-103 (2008).

66 Lee JH, Jung TY, Jung S et al. Performance status during and after radiotherapy plus concomitant and adjuvant temozolomide in elderly patients with glioblastoma multiforme. J. Clin. Neurosci. 20, 503-508 (2013).

67 Fiorentino A, Caivano R, Chiumento C et al. Comorbidity assessment and adjuvant radiochemotherapy in elderly affected by glioblastoma. Med. Oncol. 29, 3467-3471 (2012).

68 Osoba D, Brada M, Yung WK, Prados M. Health-related quality of life in patients treated with temozolomide versus procarbazine for recurrent glioblastoma multiforme. J. Clin. Oncol. 18, 1481-1491 (2000). 